\title{
The control of seed oil polyunsaturate content in the polyploid crop species Brassica napus
}

\author{
Rachel Wells • Martin Trick • Eleni Soumpourou • Leah Clissold • \\ Colin Morgan · Peter Werner · Carl Gibbard · Matthew Clarke • \\ Richard Jennaway $\cdot$ Ian Bancroft
}

Received: 6 June 2013/Accepted: 5 September 2013/Published online: 21 September 2013

(C) The Author(s) 2013. This article is published with open access at Springerlink.com

\begin{abstract}
Many important plant species have polyploidy in their recent ancestry, complicating inferences about the genetic basis of trait variation. Although the principal locus controlling the proportion of polyunsaturated fatty acids (PUFAs) in seeds of Arabidopsis thaliana is known (fatty acid desaturase 2; FAD2), commercial cultivars of a related crop, oilseed rape (Brassica napus), with very low PUFA content have yet to be developed. We showed that a cultivar of oilseed rape with lower than usual PUFA content has non-functional alleles at three of the four orthologous FAD2 loci. To explore the genetic basis further, we developed an ethyl methanesulphonate mutagenised population, JBnaCAB_E, and used it to
\end{abstract}

Electronic supplementary material The online version of this article (doi:10.1007/s11032-013-9954-5) contains supplementary material, which is available to authorized users.

R. Wells · M. Trick - E. Soumpourou ·

L. Clissold · C. Morgan · I. Bancroft ( $\square)$

John Innes Centre, Norwich Research Park,

Norwich NR4 7UH, UK

e-mail: ian.bancroft@york.ac.uk

Present Address:

L. Clissold

The Genome Analysis Centre, Norwich Research Park,

Norwich NR4 7UH, UK

P. Werner · C. Gibbard

KWS UK Ltd., 56 Church Street, Thriplow, Hertfordshire

SG8 7RE, UK identify lines that also carried mutations in the remaining functional copy. This confirmed the hypothesised basis of variation, resulting in an allelic series of mutant lines showing a spectrum of PUFA contents of seed oil. Several lines had PUFA content of $\sim 6 \%$ and oleic acid content of $\sim 84 \%$, achieving a long-standing industry objective: very high oleic, very low PUFA rapeseed without the use of genetic modification technology. The population contains a high rate of mutations and represents an important resource for research in B. napus.

Keywords Desaturation · Fatty acid biosynthesis - Brassica - Oleic acid . Polyunsaturated fatty acids $\cdot$ EMS

\footnotetext{
M. Clarke

Monsanto UK Ltd., PO Box 663, Cambridge CB1 OLD, UK

R. Jennaway

Saaten-Union UK Ltd., Rosalie Field Station, Bradley

Road, Cowlinge, Newmarket, Suffolk CB8 9HU, UK

Present Address:

I. Bancroft

Department of Biology, University of York, Heslington, York YO41 5DD, UK
} 


\section{Introduction}

Vegetable oils are an important part of the human diet, providing essential fatty acids. One of the most important oilseed crops, second in global production only to soybean, is oilseed rape (Canola). Oilseed rape is one of the crop types of the species Brassica napus. Like Arabidopsis thaliana, B. napus is a member of the Brassicacea, but unlike the simple genome of A. thaliana, that of B. napus is polyploid, which is more typical of crop species. The genetic complexity arising from polyploidy presents a barrier for the translation of knowledge from fundamental research in species such as A. thaliana into crop improvement, necessitating study of the genetic basis of traits in the crop species themselves. The lack of progress towards long-standing industry objectives in oilseed rape by traditional breeding, such as reducing the content of polyunsaturated fatty acids in rapeseed oil in order to improve its thermal stability, is a good example.

Fatty acid biosynthesis has been studied extensively in A. thaliana. The major fatty acids in seed oil are derived from the saturated fatty acid moiety stearic (which contains a backbone of 18 carbon atoms with no double bonds and is denoted C18:0). This can be elongated successively to 20 and 24 carbon fatty acids, but in modern rapeseed cultivars that pathway has been blocked by mutation of orthologues of components of the elongase complex encoded by the FAE1 locus (James et al. 1995), resulting in rapeseed oil comprising predominantly 18 carbon fatty acids. In both $A$. thaliana and rapeseed, C18:0 is sequentially desaturated to the monounsaturated fatty acid (MUFA) oleic (one double bond; denoted C18:1), then the polyunsaturated fatty acid (PUFA) linoleic (two double bonds; denoted C18:2) and finally the PUFA linolenic (three double bonds; denoted C18:3). The key control point in the biosynthesis of PUFAs in A. thaliana is the desaturase encoded by the fatty acid desaturase 2 (FAD2) locus, which catalyses the desaturation of C18:1 to C18:2 (Miquel and Browse 1992). Okuley et al. (1994) reported two T-DNA insertion lines of $A$. thaliana where disruption of the gene resulted in a considerable increase in C18:1 from $15.4 \%$ in the wild type to 37.7 and $53.5 \%$ in the fad25 and fad2-1 alleles respectively. This was accompanied by a reduction in C18 PUFA from 53 to 19.4 and $8.5 \%$ respectively, showing that inactivation of the enzyme encoded by the FAD2 locus (i.e. blocking of the desaturation pathway) results in a high oleic, low PUFA oil profile. In double mutants, in which the biosynthesis of erucic acid (C22:1) is also blocked, C18:1 accumulated to around $85 \%$ in A. thaliana (Smith et al. 2003).

The Brassica species are the closest crop relatives of the model plant A. thaliana. B. napus itself is a recently-formed allotetraploid resulting from hybridisation between the "diploid" Brassica species, B. rapa (A genome) and B. oleracea (C genome). Comparative mapping with $A$. thaliana suggest that the diploid Brassica genomes are themselves derived from a hexaploid ancestor, and the set of six related genome segments (three from $B$. rapa and three from $B$. oleracea) can be discerned clearly in B. napus (Rana et al. 2004). However, extensive interspersed gene loss has occurred during the diploidisation process following polyploidy (O'Neill and Bancroft 2000; Town et al. 2006; Yang et al. 2006; Cheung et al. 2009), so families of genes in B. napus are not necessarily present in six copies. Indeed, recent characterisation of the family of FAD2 orthologues in B. napus (denoted BnaFAD2) by Yang et al. (2012a) has confirmed that four are present within $B$. napus, as suggested previously by Scheffler et al. (1997). Family member BnaA.FAD2.b contains deletion and insertion events in its coding region, leading to mis-sense mutation and truncation, so is unlikely to be functional. Alleles of BnaA.FAD2.a that contain a 4-bp insertion in its coding region, which is expected to abolish function, resulted in an increase in C18:1 content (from 64.5 to $75 \%$ of total fatty acids). The two copies in the $\mathrm{C}$ genome (BnaC.FAD2.a and BnaC.FAD2.b) appear intact and likely to be functional. An approximation of the knock-out of the complete FAD2 gene family has been achieved by RNAi (Peng et al. 2010), which resulted in an oleic acid content of up to $85 \%$ and a reduction in PUFA to under $10 \%$. However, the stability, efficacy and precision of RNAi is unclear, particularly in polyploids. The approach is unattractive for crop improvement due to the high cost of completing the regulatory processes, but the results provide encouragement that a clearer understanding of the way in which the $B n a F A D 2$ gene family controls the trait may enable the predictive breeding of rapeseed producing very low PUFA oil.

It is generally considered that genetic diversity in $B$. napus is low, as the species arose from a limited 
number of spontaneous hybridisation events in a restricted geographic range (Mei et al. 2011), with the elite rapeseed genepool further eroded by intensive breeding with an emphasis on specific oil and seed quality traits (Hasan et al. 2006). To help overcome a lack of natural genetic variation, induced genetic variation (mutation breeding) techniques have been developed. These include the use of ionising radiation, such as X-rays or gamma rays, and chemical mutagens. Indeed, it has been estimated that over 2,000 varieties resulting from mutation breeding have been released in the last 75 years (FAO/IAEA 2010). Whilst irradiation techniques produce larger-scale genome deletions and rearrangements, chemical mutagenesis induces point mutations which may enable the identification of an allelic series of mutations. EMS produces mutations in genetic material by nucleotide substitution. The principal mechanism is via alkylation of guanine to form $\mathrm{O}^{6}$-ethylguanine, which cannot pair with cytosine but can pair with thymine. During subsequent replication, the effect is to substitute thymine for cytosine. Hence the predominant result of EMS mutagenesis is $\mathrm{C} / \mathrm{G}$ to $\mathrm{T} / \mathrm{A}$ transition changes, although occasionally $\mathrm{G} / \mathrm{C}$ to $\mathrm{C} / \mathrm{G}$ or $\mathrm{G} / \mathrm{C}$ to $\mathrm{T} / \mathrm{A}$ transversions, or $\mathrm{A} / \mathrm{T}$ to $\mathrm{G} / \mathrm{C}$ transitions occur (Krieg 1963; Greene et al. 2003). EMS has been used successfully for the development of mutagenised Brassica populations (Wang et al. 2008; Stephenson et al. 2010; Harloff et al. 2012; Himelblau et al. 2009).

Current approaches to detecting sequence variation in mutation/germplasm screens rely on methods such as Targeting Induced Local Lesions IN Genomes (TILLING) (McCallum et al. 2000; Stemple 2004) or conventional Sanger sequencing of a target amplicon. In TILLING, PCR is used to amplify an exon fragment from the target gene using pooled DNA from the individuals in a mutagenised population. The products are then melted and re-annealed before digestion with Cel1 exonuclease, which cleaves at mismatched bases in heteroduplex DNA (Oleykowski et al. 1998). The PCR fragments are then analysed using fluoro-labelled electrophoresis and mutations confirmed via PCR and sequencing from individual DNA samples. A general requirement for mutation detection involving heteroduplex analysis is the ability to develop a locus-specific PCR amplicon, which can be problematic in polyploid species, although occasionally gene families are so similar in sequence that TILLING can be conducted even with mixed amplicons (Wang et al. 2008).
Our aim was to understand the genetic basis of the control of PUFA content in rapeseed oil by functional characterisation of the family of FAD2 orthologues in B. napus. We based our study on a conventional winter oilseed rape cultivar, Tapidor (oil profile: $\sim 60 \%$ C18:1, $29 \%$ PUFAs), and a cultivar showing reduced PUFA content, Cabriolet (oil profile: $75 \%$ C18:1, $16 \%$ PUFAs). To test a hypothesis, we developed a new EMS mutagenised population of B. napus (JBnaCAB_E) from the latter and analysed the phenotypic effects in an allelic series of mutations in a specific orthologue of FAD2.

\section{Materials and methods}

Determination of homologue number and sequence of FAD2 homologues within B. napus var. Tapidor

Clones from the B. napus var. Tapidor JBnY and $\mathrm{JBnB}$ Bacterial artificial chromosome (BAC) libraries containing the BnaFAD2 genes were previously identified as described by Smooker et al. (2011). DNA from all positively hybridising clones confirmed by Southern hybridisation was prepared by standard methods (Marra et al. 1997). Alignments of the AtFAD2 sequence and BnaFAD2 sequences downloaded from Genbank produced using VectorNTi AlignX (Invitrogen) (Lu and Moriyama 2004) were used to design a degenerate forward PCR primer (ATTCCTTCCTNC TNCTNGTNCC) and a reverse primer (CAGGAGAA GTAAGGGACGAGG) within a conserved region of the gene. PCR was performed using $1 \mu \mathrm{l}$ DNA prep, $2 \mu \mathrm{l} 10 \times$ PCR buffer [500 mM KCl, $100 \mathrm{mM}$ Tris$\mathrm{HCl}$ (pH 9.0), $1 \%$ Triton X-100, $15 \mathrm{mM} \mathrm{MgCl}_{2}$ (supplied with AmplitaqTaq Gold)], $2 \mu \mathrm{l}$ forward primer $(2 \mathrm{mM}), 2 \mu \mathrm{l}$ reverse primer $(2 \mathrm{mM}), 1.3 \mu \mathrm{l}$ dNTPs ( $2 \mu \mathrm{M}$ Invitrogen Cat. No. 10297-018), $0.2 \mu \mathrm{l}$ Amplitaq Gold $5 \mathrm{u} / \mu \mathrm{l}$ (Applied Biosystems Cat. No.

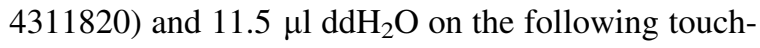
down cycle: $94{ }^{\circ} \mathrm{C}$ for $5 \mathrm{~min}, 15$ cycles of $94{ }^{\circ} \mathrm{C}$ for $30 \mathrm{~s}, 64{ }^{\circ} \mathrm{C}$ for $30 \mathrm{~s}\left(-1{ }^{\circ} \mathrm{C}\right.$ per cycle $)$ and $72{ }^{\circ} \mathrm{C}$ for $30 \mathrm{~s}, 30$ cycles of $94{ }^{\circ} \mathrm{C}$ for $30 \mathrm{~s}, 53{ }^{\circ} \mathrm{C}$ for $30 \mathrm{~s}$ and $72{ }^{\circ} \mathrm{C}$ for $30 \mathrm{~s}$, then $72{ }^{\circ} \mathrm{C}$ for $7 \mathrm{~min}$, and store at $8{ }^{\circ} \mathrm{C}$. To determine the individual homologues of $F A D 2$ present within the clones, sequencing was performed on $1 \mu \mathrm{l}$ of PCR product cleaned up by isopropanol precipitation using the BigDye Terminator v3.1 cycle 
sequencing kit (Applied Biosystems Cat. No. 100 reactions 4337455) according to the manufacturer's instructions (Applied Biosystems). Individual clone sequences were again compared using AlignX and divided into homologue groups. To obtain the sequence of the complete BnaFAD2 homologues, one clone per group was chosen and sequenced by external commercial service providers (JIC Genome Laboratory; GATC-Biotech in Konstanz, Germany; Beijing Genomics Institute). To confirm that no further homologues of BnFAD2 were present within B. napus, cloning of PCR product from a conserved forward primer (CCTCGTCCCTTACTTCTCCTG) and the reverse primer previously used to screen the BAC clones amplified on a standard $50{ }^{\circ} \mathrm{C}$ PCR cycle, was performed on genomic Tapidor DNA using the pGEM-T Easy vector system kit according to the manufacturer's instructions (Promega Cat. No. A1360). Colony PCR and sequencing using the vector SP6 and T7 primers was then performed for 11 clones and sequences aligned against the previously identified homologues.

\section{Mapping of BnaFAD2 homologues}

Three of the BnaFAD2 homologues were mapped as previously described by Smooker et al. (2011). The chromosome allocation of the remaining homologue was implied by homology to the $B$. rapa genome sequence (Wang et al. 2011).

Determination of sequence of BnaFAD2

homologues within B. napus var. Cabriolet

A combination of specific and conserved primers designed from the Tapidor reference sequence was used to obtain the gene sequence from Cabriolet following the touchdown PCR protocol described above, with the first touchdown cycle starting at $63{ }^{\circ} \mathrm{C}$. Primers are detailed in Supplemental Table 1. Primer combinations for amplifying $\sim 1,000 \mathrm{bp}$ of each homologue are given in Supplemental Table 2.

Expression analysis of BnaFAD2 homologues

Ten seeds of Tapidor and Cabriolet were sown, pricked out into individual $6-\mathrm{cm}$ pots at the two-leaf stage and, at the four-leaf stage, vernalised at $4{ }^{\circ} \mathrm{C}$ for 6 weeks. Following vernalisation, plants were transferred to a glasshouse at $12-18{ }^{\circ} \mathrm{C}$ with $16-\mathrm{h}$ day length, and repotted into 1-L pots. RNA was extracted from developing seed 45 DPA (a stage at which Bna.FAD2 gene expression is expected to be high) using the RNeasy plant mini kit (Qiagen). Buffer RLC (containing guanidine hydrochloride) was substituted for buffer RLT due to the secondary metabolites present within the seed. cDNA was synthesised using the Superscript III First Strand Synthesis System for RT-PCR (Invitrogen Cat. No. 18080-051). PCR and cloning of RTPCR product was performed as for the genomic samples, detailed previously.

Transcriptome sequencing of Tapidor and Cabriolet seed

As amplification could not be achieved for homologue BnaC.FAD2.a within Cabriolet, transcriptome sequencing, which does not rely on homologue amplification, was performed on the RNA from developing seed 45 days after pollination (DAP) using the 80-bp Illumina RNA-seq GAII platform by TGAC. Reads were aligned against the Tapidor reference sequences and data viewed using Tablet Next Generation Sequence Assembly Visualisation software (Milne et al. 2010).

Mutagenesis of B. napus var. Cabriolet

EMS mutagenesis was carried out on $\sim 33,000$ seeds of B. napus var. Cabriolet. Five treatments of $150 \mathrm{ml}$ of 0.2, 0.4, 0.6, 0.8 and $1 \%$ EMS (Sigma-Aldrich Cat. No. M0880) in $0.02 \%$ Tween 20 solution (SigmaAldrich Cat. No. TS700-500ML) were performed on $30 \mathrm{~g}(\sim 6,400)$ seed each. Treatments of $10 \mathrm{ml} 0.02 \%$ Tween 20 and $10 \mathrm{ml} 2 \%$ EMS were performed on $2.5 \mathrm{~g}(\sim 500)$ seed as negative and positive controls respectively. Seed treatment tubes were placed in a rotating box and set to turn slowly overnight (18 h). EMS decontamination of the seed was achieved by performing 10 washes of $150 \mathrm{ml} 0.02 \%$ Tween 20 for 20 min per wash, turning slowly. After the final wash, seeds were transferred to KWS UK for sowing at a density of 500 seeds per $348 \mathrm{~mm} \times 220 \mathrm{~mm}$ tray. Trays were kept at $4{ }^{\circ} \mathrm{C}$ for 2 days to stratify before transferring to glasshouse at $18{ }^{\circ} \mathrm{C}$ with $16 \mathrm{~h}$ light. Emergence was scored 7 days after sowing. 10,080 lines were grown on for seed from treatments predicted to contain a good mutation load but still 
maintain viability: $4,5780.4 \%$ EMS lines, 4,410 $0.6 \%$ EMS lines and 1,092 $0.8 \%$ EMS lines.

$\mathrm{M}_{2}$ population growth

Two 17-cm spaced double rows of seed for each $\mathrm{M}_{2}$ line were drilled for 7,684 lines (2,738 0.4\% EMS, 3,873 $0.6 \%$ EMS and 1,073 $0.8 \%$ EMS) distributed between two sites within the UK (Newmarket and Cambridge) and a site in France (Boissay). One plant per double row was labelled, bagged for seed production and leaf material was collected. DNA isolation was carried out using the DNeasy Plant 96 Qiagen Kit for 96 samples following the manufacturer's instructions (Qiagen, UK). $\mathrm{M}_{3}$ seed was collected, threshed and deposited within the John Innes Centre seed-store $\left(1.5^{\circ} \mathrm{C}, 7-10 \%\right.$ relative humidity) to ensure their long-term viability.

Screening of the $\mathrm{M}_{2}$ population for BnaC.FAD2a mutations

A 1,212-bp BnaC.FAD2.b specific amplicon was amplified from 3000 seeds of the 0.8 and $0.6 \%$ treated EMS line using the primers GTCTCCTCCCT CCAAAAAGT and CAAGACGACCAGAGACAGC with the standard PCR recipe detailed for homologue sequencing on the following cycle: $94{ }^{\circ} \mathrm{C}$ for $5 \mathrm{~min}$, 35 cycles of $94{ }^{\circ} \mathrm{C}$ for $30 \mathrm{~s}$ (ramp $0.5{ }^{\circ} \mathrm{C} / \mathrm{s}$ ), $57{ }^{\circ} \mathrm{C}$ for $30 \mathrm{~s}$ (ramp $0.5{ }^{\circ} \mathrm{C} / \mathrm{s}$ ) and $72{ }^{\circ} \mathrm{C}$ for $1 \mathrm{~min}$ (ramp $0.5^{\circ} \mathrm{C} / \mathrm{s}$ ), then $72{ }^{\circ} \mathrm{C}$ for $10 \mathrm{~min}$, and store at $8{ }^{\circ} \mathrm{C}$. Unincorporated primer and dNTPs were removed from $10 \mu \mathrm{l}$ of the product by SAPEXO treatment [ $1 \mu \mathrm{l}$ shrimp alkaline phosphotase (Roche Cat. No. 04898133001) and $0.5 \mu \mathrm{l}$ exonuclease 1 (EXO) (GE Healthcare Cat. No. E700732)], samples incubated at $37^{\circ} \mathrm{C}$ for $30 \mathrm{~min}$, and denatured at $80{ }^{\circ} \mathrm{C}$ for $10 \mathrm{~min}$ before sequencing was performed on $1 \mu \mathrm{l}$ of product using the BigDye Terminator v3.1 cycle sequencing kit (Applied Biosystems Catalogue number 100 reactions 4337455) according to the manufacturer's instructions. Mutations were scored by electropherogram alignment to wild-type Cabriolet using MutationSurveyor v2.61 software (SoftGenetics, State College, PA, USA) (Dong and Yu 2011).

Growth and phenotyping of $\mathrm{M}_{3}$ lines

$12 \mathrm{M}_{3}$ seeds were sown for each line at KWS and DNA prepared from a young leaf for each line as for the $M_{2}$.
Sequencing of BnaC.FAD2.a PCR product as detailed above was performed to select homozygous and wildtype out-segregant lines for phenotyping. Multiple homozygotes of each line and outsegregant examples, where available, were grown in long-day glasshouse conditions (16-h photoperiod and $18{ }^{\circ} \mathrm{C} / 14{ }^{\circ} \mathrm{C}$ day/ night) in a randomised split block design. A maximum of twelve replicate plants of three lines, Tapidor, Cabriolet and V141, were used as standard controls. Phenotyping for fatty acid profile was performed by gas chromatography (conducted at KWS) using standard industry protocols.

\section{Results}

Characterisation of the BnaFAD2 family in Tapidor and Cabriolet

BAC clones derived from genomic DNA of B. napus var. Tapidor that had been identified previously by Smooker et al. (2011) as containing sequences that hybridised to $F A D 2$-specific probes were sequenced. The results confirmed the presence of four orthologues in B. napus, as suggested by Yang et al. (2012b). Three copies had been positioned previously by linkage mapping (BnaA.$F A D 2 . a$ on linkage group A5, BnaC.FAD2.b on $\mathrm{C} 1$ and BnaC.FAD2.a on C5; Smooker et al. 2011). We positioned the fourth (BnaA.FAD2.b) on linkage group A1 based on sequence similarity with the $B$. rapa genome sequence (Wang et al. 2011). These positions corresponded to those previously indicated, based on restriction fragment length polymorphism mapping, by Scheffler et al. (1997). Alignment of the coding regions of the genes showed a high level of sequence conservation between the pairs of homoeologous genes, i.e. those on A1 and C1 (95\%), and those on A5 and C5 (96 \%) (see Supplemental Figure 1). The Tapidor allele of BnaA.FAD2.b, as with the allele in the Chinese germplasm characterised by Yang et al. (2012b), contained deletion and insertion events predicted to result in frame shifts and a truncated protein. The other three copies are predicted to encoded full-length proteins of 383 amino acids (see Supplemental Figure 2). All copies were found to be expressed in the seed, using both RT-PCR and mRNAseq (data not shown).

Oil from the rapeseed variety Cabriolet contains $\sim 75 \%$ oleic and $16 \%$ PUFAs, compared with $\sim 60 \%$ oleic and $29 \%$ PUFAs in variety Tapidor, 
suggesting that fewer FAD2 orthologues may be functional in Cabriolet than in Tapidor. We used locus-specific PCR amplification and sequencing to characterise the family of $F A D 2$ orthologues in Cabriolet, with primer design based on the sequences of the Tapidor alleles. Sequence analysis revealed the same frame shifts to be present in the Cabriolet allele of BnaA.FAD2.b. A 1-bp deletion was identified in the Cabriolet allele of BnaA.FAD2.a, predicted to result in a frame shift and a truncated protein. We were unable to amplify BnaC.FAD2.b from Cabriolet and could detect no expression by mRNAseq analysis of RNA from seeds 45 DAP, suggesting deletion of this gene. Thus only BnaC.FAD2.a appears to encode a functional protein in Cabriolet.

Development of an EMS-induced mutation population

As we wished to test the hypothesis that BnaC.FAD2.a is the only functional copy of the gene family in Cabriolet, we induced mutations in this genetic background. Approximately 33,000 seeds of variety Cabriolet were treated with a range of doses of EMS and from them a population, which we called the JBnaCAB_E population, was developed. Observation of seedling emergence from the treated seeds showed that treatment with increasing EMS concentration generally resulted in reduced seeding emergence and vigour, with $2 \%$ EMS resulting in no viable seedlings. Treatments with $0.4,0.6$ and $0.8 \%$ EMS were chosen to be grown on to produce the population. Emergence, fertility and seed number details are shown in Supplemental Table 3 . Approximately 20 seeds were collected from each of these $\mathrm{M}_{1}$ generation plants.

The $\mathrm{M}_{2}$ generation was grown, with subsets of the population grown each at one of three sites (Thriplow and Cowlinge in UK and Boissay in France). All of the $\sim 20$ seeds from each $\mathrm{M}_{1}$ plant were sown, together, but only one plant was bagged for seed collection. Duplicate leaf tissue samples were taken from the bagged plants and DNA was prepared from one sample. The resulting population (as packets of $\mathrm{M}_{3}$ generation seeds derived from individual $\mathrm{M}_{2}$ generation plants) comprised 1,678, 3,441 and 1,037 lines resulting from treatments with $0.4,0.6$ and $0.8 \%$ EMS, respectively. Few phenotypic abnormalities were observed within the population and no visible differences were detected between different treatment levels.
Induced mutation of BnaC.FAD2.a

To search for mutations in BnaC.FAD2.a, we designed a 1,212-bp locus-specific PCR amplicon. After checking specificity, we first assessed the rates of mutations induced by the various EMS treatments by amplification using the DNA prepared from leaf samples as template. To do this, we amplified the 1,212-bp region of BnaC.FAD2.a and sequenced the PCR products (capillary sequencing). This revealed mutation rates of $1.3 \%$ (4/302), $2.6 \%$ (7/274) and $4.9 \%$ (14/288) for $0.4,0.6$ and $0.8 \%$ EMS treatments, respectively, showing the expected increasing mutation load with increasing severity of EMS treatment. To complete the screening of the population, further lines (drawn from 0.6 to $0.8 \%$ EMS treatment) were screened by amplification and sequencing of the 1,212-bp region of BnaC.FAD2.a. In all, a subset of $\sim 3,000$ lines was screened. Where mutations were identified in a line, 12 seeds from the line $\left(\mathrm{M}_{3}\right.$ generation) were sown and tested for the presence of the detected mutation by PCR amplification and sequencing using DNA purified from leaf samples taken from individual seedlings. The sequence trace files were analysed, enabling robust assessment of whether mutations were present in homozygous state (the trace file shows a clean call of the altered base) or heterozygous state (approximately half-height peaks are present in the traces at the position of the altered base). In total, 102 mutations were identified and confirmed in BnaC.FAD2.a. Of these, five are predicted to result in the introduction of premature stop codons, 52 are predicted to result in amino acid substitutions and 45 are predicted to be silent. All mutations detected are shown in Supplemental Table 4.

Phenotypic analysis of lines with mutations induced in BnaC.FAD2.a

The subset of lines containing non-silent homozygous BnaC.FAD2.a mutations was assessed for effects on oil composition. To do this, the $\mathrm{M}_{3}$ plants used for confirmation of mutations were grown on in an unbalanced randomised block design under glasshouse conditions. The number of homozygous mutant individuals available varied between lines, from one to six (depending upon segregation in the $\mathrm{M}_{2}$ generation; hence the necessity of an unbalanced design). Most lines were grown on to maturity, including 
Fig. 1 Distribution of oleic acid and 18-carbon polyunsaturated fatty acid composition of seeds in mutant and control lines. a Oleic acid (C18:1), b total 18-carbon polyunsaturated fatty acid (Total C18 PUFA) in seeds of $B$. napus variety Cabriolet $(\mathrm{Cab})$,

BnaA.FAD2.a mutated lines (Mutant), outsegregant lines (Null) and a commercial high oleic, low linolenic line (V141)
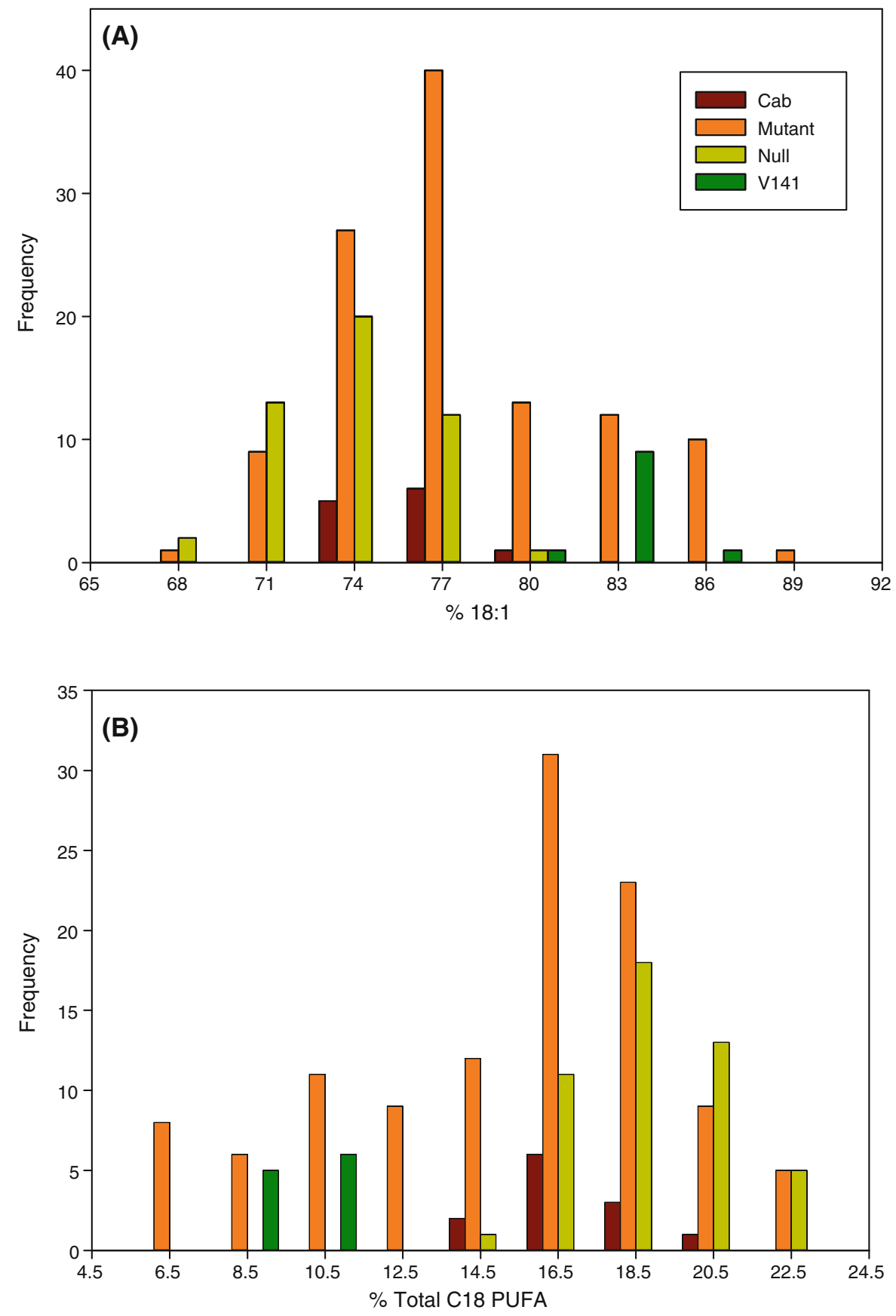

heterozygous and a proportion of outsegregant wild types for lines with confirmed mutations. Control lines included Tapidor, as a representative of conventional rapeseed ( $60 \% \mathrm{C} 18: 1,29 \%$ PUFAs), Cabriolet as the background for mutation $(\sim 75 \% \mathrm{C} 18: 1,16 \%$ PUFAs) and V141, as a representative of the state-ofthe-art high oleic lines produced by commercial breeding ( 79\% C18:1, $10 \%$ PUFAs).
Analysis of the Cabriolet controls showed no lineby-block interactions and no significant differences for content of any fatty acid. Analysis of outsegregant lines not inheriting BnaC.FAD2.b mutations showed no line-by-block interactions but did show modest, but significant $(P<0.05$ unless stated $)$, differences for C16:0, C16:1 $(P<0.01), \quad \mathrm{C} 18: 1, \quad \mathrm{C} 18: 3, \mathrm{C} 20: 2$ $(P<0.01)$ and $\mathrm{C} 24: 1$. The greater variability of 
outsegregants than the original genotype is likely a consequence of the very large number of background mutations segregating in lines. Similar variability would likely be superimposed on effects arising from mutation of BnaC.FAD2.a. As shown in Fig. 1 (for C18:1 and PUFA content) and Supplemental Figure 3 (for all other major fatty acids measured), the range of phenotypes for individual plants homozygous for mutation of BnaC.FAD2.a exceeds that for outsegregants, with notable skew towards higher C18:1 and lower PUFA content.

A breakdown of mean composition for the fatty acids quantified is shown in Table 1 for 36 lines showing non-silent mutations of BnaC.FAD2.a, along with those for Tapidor, Cabriolet and V141. Many mutations result in little or no difference in the content of C18:1 or PUFAs. However, the mutation predicted to result in a stop codon and premature termination of the protein encoding the desaturase enzyme, along with seven further mutations, resulted in marked decreases in PUFAs, to $\sim 6 \%$ (compared with $\sim 16 \%$ for Cabriolet), and marked increase in C18:1, to $\sim 84 \%$ (compared with $\sim 75 \%$ for Cabriolet). We can exclude the possibility of the observed phenotype being caused by mutation of a gene other than BnaC.FAD2.a (i.e. a second site mutation) as it occurs in many independently-derived lines, even any two of which are highly unlikely to share mutations of another locus. The results of the analysis of the oil composition of lines heterozygous for these eight mutations are shown in Supplemental Figure 4. Eight other mutations resulted in intermediate phenotypes (ranges $8.5-12 \%$ PUFAs; 79-82 \% C18:1). The protein sequence of BnaC.FAD2.a showing the positions of all mutations is shown in Fig. 2. The mutations induced in BnaC.FAD2.a, as expected, had no impact on either content of saturated fatty acids (C14:0, C16:0, C18:0) or very long chain fatty acids (C20-24). In contrast to the fatty acid composition of seeds of V141, which contain around twice as much C18:2 as C18:3 (presumably caused by mutation of another locus, perhaps an orthologue of FAD3), the reduction in total PUFA content in mutations of BnaC.FAD2.a is accompanied by an increase in the proportion of $\mathrm{C} 18: 3$ to $\mathrm{C} 18: 2$, resulting in around twothirds the amount of C18:2 as C18:3 in BnaC.FAD2.a mutant lines with PUFA content similar to, or lower than, that of V141.

\section{Discussion}

Development of the JBnaCAB_E population

We developed an EMS mutagenised population of B. napus consisting of $\sim 5500$ lines comprising subpopulations with three different severities of EMS treatment. Despite the high mutation loads, estimated as $1.3,2.6$ and $4.9 \%$ for $0.4,0.6$ and $0.8 \%$ EMS treatments, respectively, we observed remarkably few visible phenotypes. In contrast, Stephenson et al. (2010) saw a wide range of visible phenotypes in a $B$. rapa TILLING population treated with $0.3 \%$ EMS and estimated to carry a $2.7 \%$ mutation load. The difference is likely due to the much greater genetic redundancy in $B$. napus, which is a recently-formed allotetraploid in which one genome was inherited from a $B$. rapa progenitor and the other from a $B$. oleracea progenitor. This observation does not mean that there is no upper limit to the EMS treatment that $B$. napus can withstand. For example, treatment with $2 \%$ EMS resulted in failure of seeds to germinate and establish. In addition, the higher level of background mutations in more severely mutated material may require more extensive backcrossing to re-establish vigour. Nevertheless, the JBnaCAB_E population represents an excellent resource for the genetic analysis of traits in a winter oilseed rape crop type of B. napus.

Functional characterisation of the BnaFAD2 family

Our analysis of the BnaFAD2 gene family in variety Cabriolet revealed the presence of only one functional member of the family, BnaC.FAD2.a. As we are aware of no reports of additivity for $F A D 2$ family members or co-dominance of mutant alleles, we hypothesised that the reduced PUFA phenotype in Cabriolet compared with conventional rapeseed cultivars, such as Tapidor, may be the result of loss of function at three loci revealing the reduced potency of the enzyme encoded by the one remaining locus, BnaC.FAD2.a. To test whether enzyme function could be modulated in this way, we produced a large allelic series of mutations of this locus by severe EMS treatment of the Cabriolet genotype. The result was, indeed, lines exhibiting a range of phenotypes, with the most severely affected having the phenotype $\sim 84 \%$ C18:1, $\sim 6 \%$ PUFAs. This phenotype matches that found to be the result of 


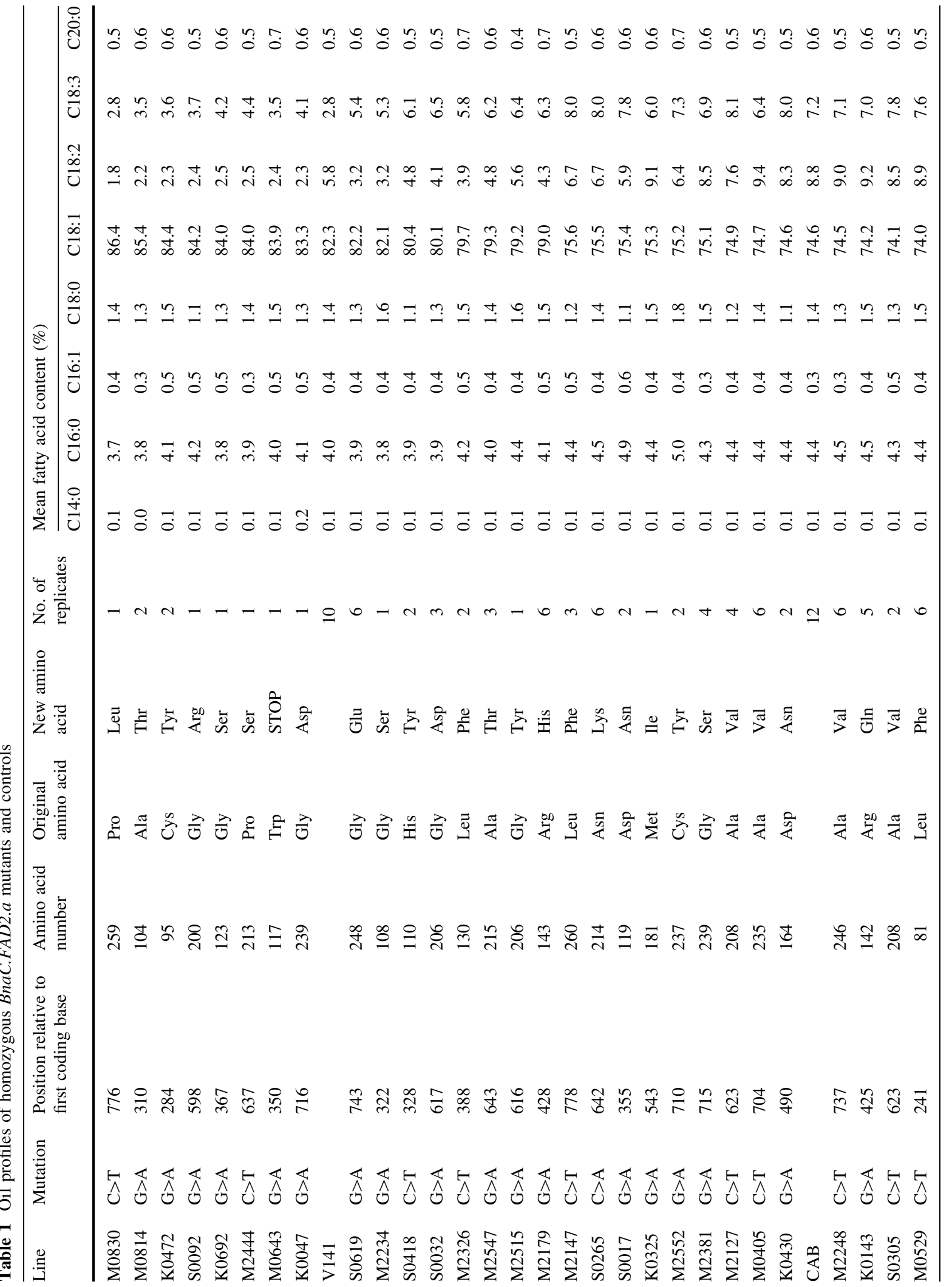




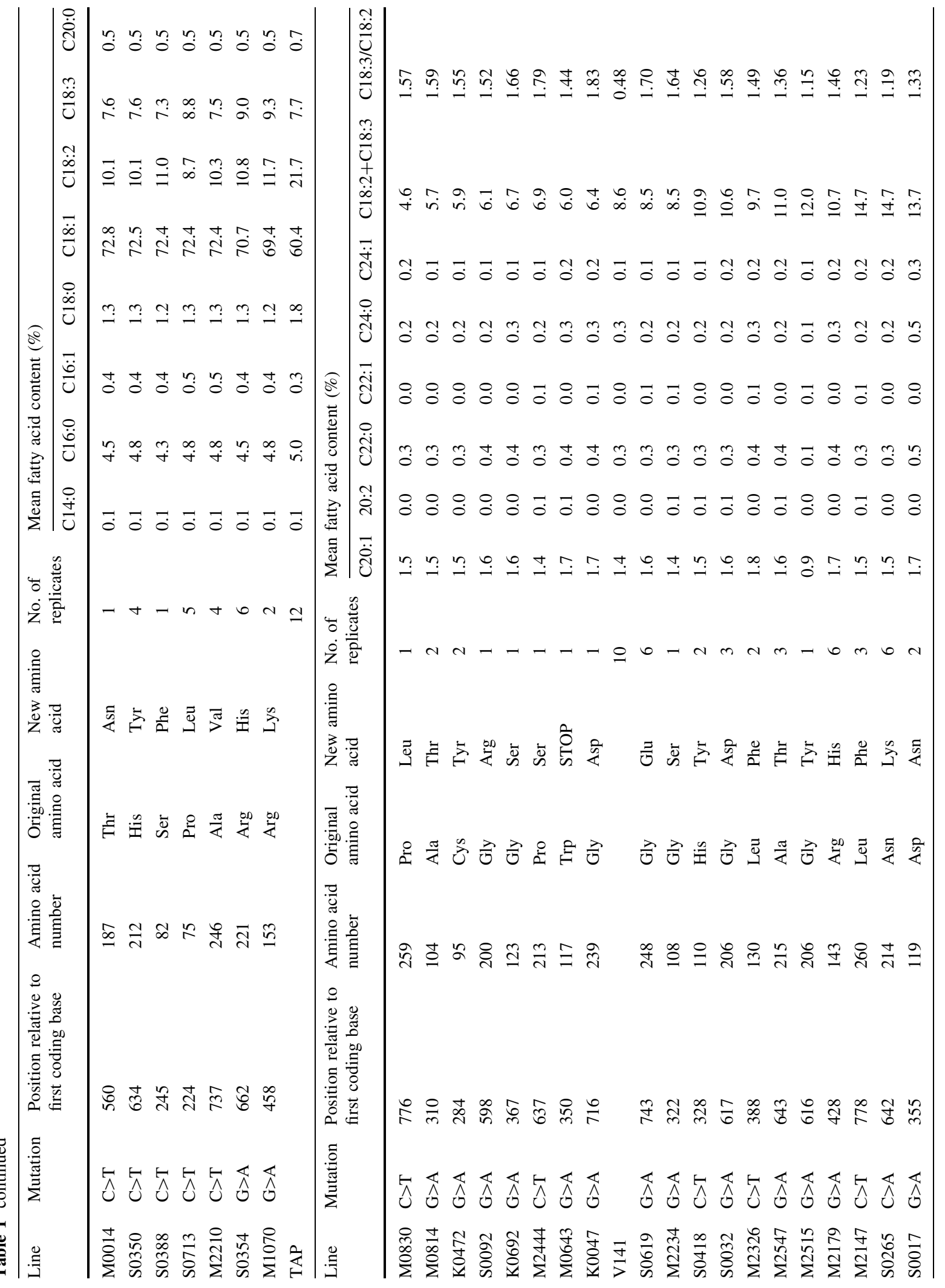




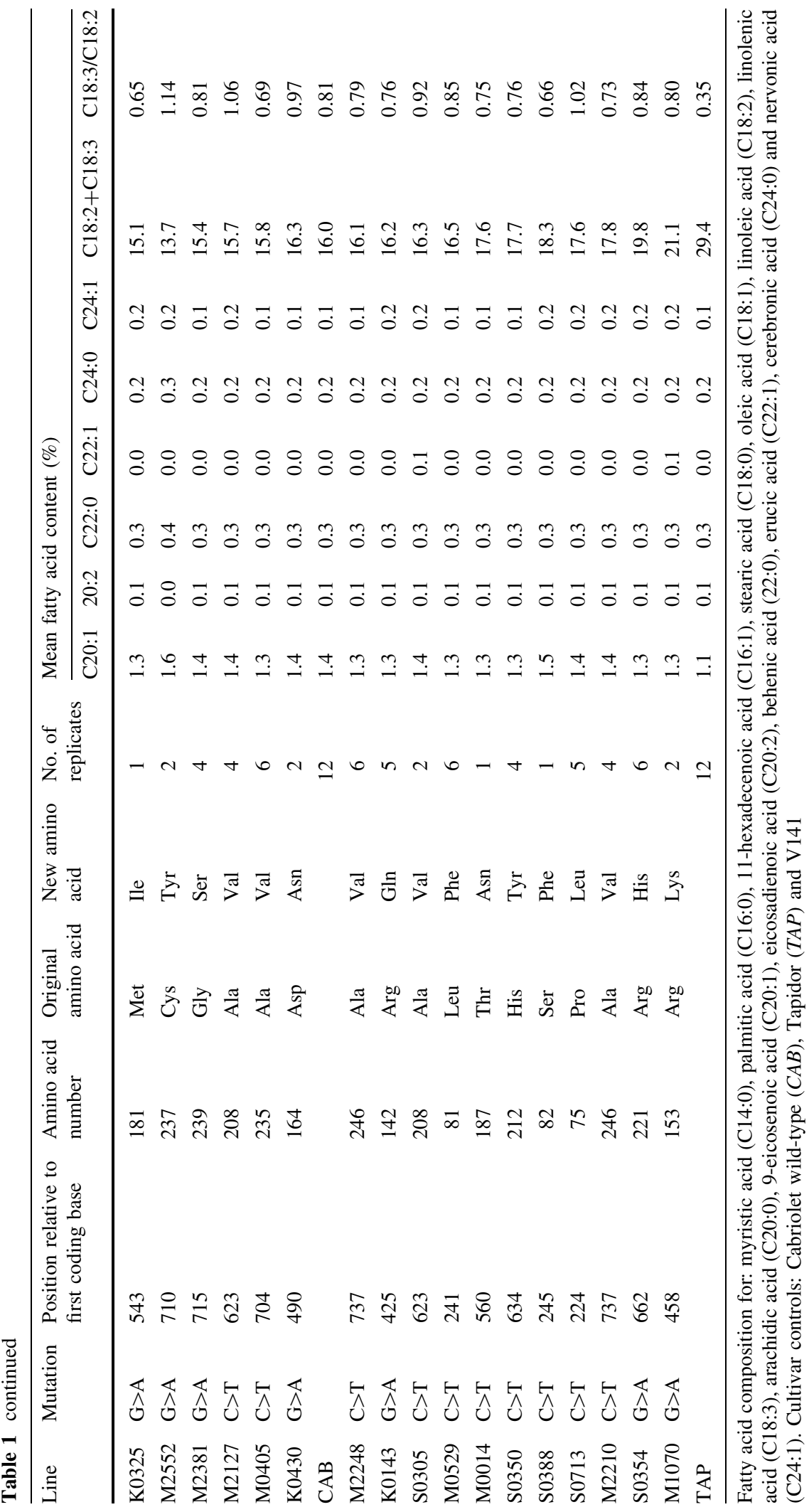


MGAGGRMQVSPPSKKSETDTIKRVPCETPPFTVGELKKAIPPH CFKRSIPRSFSYLIWDIIIASCFYYVATTYFPLLPHPLSYFAW PLYWACQGCVLTGVWVIAHEC $\mathrm{H}$ AFSDYQWIDDTVGLIFHSF ILVPYFSWKYSHRRHHSNTGSLERDEVFVPKKKSDIKWYGLYL NNPLGRTVMLTVQFTLGWPLYLAFNVSGRPYDG FACHFHPNA PIYNDRERLQIYISDAGILAVCYGLFRYAAAQ VASMVCFYGV PLLIVNGFLVLITYLQHTHPSLPHYDSSEWDWLRGALATVDRD YGILNKVFHNITDTHVAHHLFSTMPHYHAMEATKAIKPILGEY YQFDGTPVVKAMWREAKECIYVEPDRQGEKKGVFWYNNKL *

Fig. 2 Phenotypic effects of altering amino acids in the desaturase encoded at BnaC.FAD2.a. Red mutation results in polyunsaturated fatty acid content below $7 \%$ and oleic acid content over $80 \%$. Blue mutation results in fatty acid composition similar to wild type. Orange mutation results in intermediate fatty acid composition. The amino acid position mutated to a stop codon in line M0643 is boxed

RNAi knock-down of the gene family in B. napus (Peng et al. 2010), with the remaining PUFAs likely originating from the plastidial biosynthesis pathway. In addition, there are several genotypes with intermediate oil compositions, consistent with further impairment (but not abolition) of function. Thus our results confirm that B. napus contains four orthologues of $F A D 2$, that the breeders in achieving the reduced PUFA phenotype observed in Cabriolet have brought together knock-out mutations of three copies and the final functional copy is susceptible to modulation of function by EMS mutagenesis.

We have produced a series of mutations that enable assessment of the functional significance of specific amino acids in the linoleate desaturase encoded by BnaC.FAD2.a. Before these assessments can be performed rigorously, it will be necessary for the lines to undergo an extensive program of backcrossing to reduce background mutations that might affect the phenotype. In the meantime, however, our results provide indications of those that are likely to be critical (i.e. those for which we have detected a change in oil composition) or unlikely to be so important (i.e. those at which mutations result in little or no change in oil composition). None of the lines produced detectable quantities of unusual fatty acids.

\section{Novel rapeseed oils}

By targeting specifically BnaC.FAD2.a in variety Cabriolet, we have produced an allelic series of lines with variation in the proportions of C18:1 and PUFAs in their oil. When produced on a sufficient scale, these novel types of rapeseed oil may have important uses.

The principal effect on the physical properties of reducing the PUFA content of rapeseed oil is anticipated to be an increase in its thermal stability, as saturated fatty acids (SFA) and MUFAs are more stable than PUFAs. This will make it more suitable not only for high-temperature cooking, but also for hightemperature industrial applications, such as engine lubricants and hydraulic fluids, as a substitute for mineral oil. It is noteworthy that even in the lines with the lowest PUFA content, the SFA content of the oil has not increased (which is good as SFAs tend to increase the temperature at which oils begin to set) and that $\mathrm{C} 18: 3$ has been reduced only modestly (which is good as C18:3 is linked to cold tolerance).

Dietary studies into the benefits of PUFAs in the diet are confounded by a lack of suitable variation within a type of vegetable oil. The allelic series produced by mutation of BnaC.FAD2.a could be used to produce a panel of rapeseed oils with varying PUFA contents. Lines with very similar C18:1 and overall PUFA contents to V141, such as S0619, could be used in combination with oil from V141 to test the effects of varying proportions of $\mathrm{C} 18: 2$ and $\mathrm{C} 18: 3$ in the diet.

Although the very high C18:1 phenotype that we have produced by mutation could also be produced by RNAi, there are important advantages associated with genetic variation induced by mutation breeding over that induced by genetic modification (GM) technology. In particular, the cost of completing the regulatory process for GM crops is prohibitive. There are no such costs for material produced by mutation breeding. The RNAi approach, particularly if seed-specific targeting of the silencing was incorporated, may reduce any tendency to cold susceptibility. However, the high oleic lines we have produced grow successfully over the winter in UK conditions, so cold susceptibility appears not to be a problem.

\section{Conclusions}

The genome redundancy arising from polyploidy makes the genetic study of traits, necessary for predictive breeding, challenging in many crop species. Our study demonstrates that quantitative reduction in PUFA content of oil in oilseed rape variety Cabriolet is 
the consequence of breeders selecting and combining knock-out alleles, as has been observed for other traits in rapeseed that have been the focus of breeding, such as seed glucosinolate content (Harper et al. 2012). The phenotypic values of such traits can now be extended in a predictive way in winter oilseed rape, by targeting further relevant loci using the JBnaCAB_E population. By thoroughly understanding the genetic basis of PUFA content of rapeseed oil, we successfully knocked out the remaining functional orthologue of $F A D 2$, resulting in an oil composition very high in C18:1 and very low in PUFAs. By achieving it in this way, the newly-developed crop type can be cultivated for applications such as renewable alternatives to mineral oils as lubricants without the regulatory costs associated with transgenic approaches.

Acknowledgments This work was sponsored by BBSRC through the Renewable Materials LINK programme (LK0843) and by HGCA (RD-2007-3356). We would like to thank Frederic Beaudoin for the analysis of mutant lines for potential novel fatty acids.

Open Access This article is distributed under the terms of the Creative Commons Attribution License which permits any use, distribution, and reproduction in any medium, provided the original author(s) and the source are credited.

\section{References}

Applied Biosystems (2002) BigDye ${ }^{\mathrm{TM}}$ Terminator v3.1 Ready Reaction Cycle Sequencing Kit Protocol

Cheung F, Trick M, Drou N, Lim YP, Park J-Y, Kwon S-J, Kim J-A, Scott R, Pires JC, Paterson AH, Town C, Bancroft I (2009) Comparative analysis between homoeologous genome segments of Brassica napus and its progenitor species reveals extensive sequence-level divergence. Plant Cell Online 21(7):1912-1928. doi:10.1105/tpc.108.060376

Dong C, Yu B (2011) Mutation surveyor: an in silico tool for sequencing analysis. Methods Mol Biol 760:223-237. doi:10.1007/978-1-61779-176-5_14

FAO/IAEA (2010) The FAO/IAEA database of mutation enhanced technologies for agriculture (META). http:// mvgs.iaea.org/

Greene EA, Codomo CA, Taylor NE, Henikoff JG, Till BJ, Reynolds SH, Enns LC, Burtner C, Johnson JE, Odden AR, Comai L, Henikoff S (2003) Spectrum of chemically induced mutations from a large-scale reverse-genetic screen in Arabidopsis. Genetics 164(2):731-740

Harloff H-J, Lemcke S, Mittasch J, Frolov A, Wu J, Dreyer F, Leckband G, Jung C (2012) A mutation screening platform for rapeseed (Brassica napus L.) and the detection of sinapine biosynthesis mutants. Theor Appl Genet 124(5): 957-969. doi:10.1007/s00122-011-1760-Z
Harper AL, Trick M, Higgins J, Fraser F, Clissold L, Wells R, Hattori C, Werner P, Bancroft I (2012) Associative transcriptomics of traits in the polyploid crop species Brassica napus. Nat Biotechnol 30(8):798-802. http://www.nature. com/nbt/journal/v30/n8/abs/nbt.2302.html\#supplementaryinformation

Hasan M, Seyis F, Badani AG, Pons-Kühnemann J, Friedt W, Lühs W, Snowdon RJ (2006) Analysis of genetic diversity in the Brassica napus L. gene pool using SSR markers. Genet Resour Crop Evol 53(4):793-802. doi:10.1007/ s10722-004-5541-2

Himelblau E, Gilchrist E, Buono K, Bizzell C, Mentzer L, Vogelzang R, Osborn T, Amasino R, Parkin IP, Haughn G (2009) Forward and reverse genetics of rapid-cycling Brassica oleracea. Theor Appl Genet 118(5):953-961. doi:10.1007/s00122-008-0952-7

James DW, Lim E, Keller J, Plooy I, Ralston E, Dooner HK (1995) Directed tagging of the Arabidopsis FATTY ACID ELONGATION1 (FAE1) gene with the maize transposon activator. Plant Cell Online 7(3):309-319. doi:10.1105/tpc. 7.3.309

Krieg DR (1963) Ethyl methanesulfonate-induced reversion of bacteriophage T4rII mutants. Genetics 48(4):561-580

Lu G, Moriyama EN (2004) Vector NTI, a balanced all-in-one sequence analysis suite. Brief Bioinform 5(4):378-388. doi:10.1093/bib/5.4.378

Marra MA, Kucaba TA, Dietrich NL, Green ED, Brownstein B, Wilson RK, McDonald KM, Hillier LW, McPherson JD, Waterston RH (1997) High throughput fingerprint analysis of large-insert clones. Genome Res 7(11):1072-1084. doi:10.1101/gr.7.11.1072

McCallum CM, Comai L, Greene EA, Henikoff S (2000) Targeting induced local lesions IN genomes (TILLING) for plant functional genomics. Plant Physiol 123(2):439-442. doi:10.1104/pp.123.2.439

Mei J, Fu Y, Qian L, Xu X, Li J, Qian W (2011) Effectively widening the gene pool of oilseed rape (Brassica napus L.) by using Chinese $B$. rapa in a 'virtual allopolyploid' approach. Plant Breed 130(3):333-337. doi:10.1111/j. 1439-0523.2011.01850.x

Milne I, Bayer M, Cardle L, Shaw P, Stephen G, Wright F, Marshall D (2010) Tablet-next generation sequence assembly visualisation. Bioinformatics 26(3):401-402

Miquel M, Browse J (1992) Arabidopsis mutants deficient in polyunsaturated fatty-acid synthesis-biochemical and genetic-characterization. J Biol Chem 267:1502-1509

Okuley J, Lightner J, Feldmann K, Yadav N, Lark E, Browse J (1994) Arabidopsis FAD2 gene encodes the enzyme that is essential for polyunsaturated lipid synthesis. Plant Cell Online 6(1):147-158. doi:10.1105/tpc.6.1.147

Oleykowski C, Bronson Mullins C, Godwin A, Yeung A (1998) Mutation detection using a novel plant endonuclease. Nucleic Acids Res 26(20):5

O’Neill CM, Bancroft I (2000) Comparative physical mapping of segments of the genome of Brassica oleracea var. albogalabra that are homeologous to sequenced regions of chromosome 4 and 5 of Arabidopsis thaliana. Plant $\mathbf{J}$ 23(2):233-243

Peng Q, Hu Y, Wei R, Zhang Y, Guan C, Ruan Y, Liu C (2010) Simultaneous silencing of FAD2 and FAE1 genes affects both oleic acid and erucic acid contents in Brassica napus 
seeds. Plant Cell Rep 29(4):317-325. doi:10.1007/s00299010-0823-y

Rana D, Boogaart T, O'Neill CM, Hynes L, Bent E, Macpherson L, Park JY, Lim YP, Bancroft I (2004) Conservation of the microstructure of genome segments in Brassica napus and its diploid relatives. Plant J 40(5):725-733. doi:10.1111/j. 1365-313X.2004.02244.X

Scheffler JA, Sharpe AG, Schmidt H, Sperling P, Parkin IAP, Lühs W, Lydiate DJ, Heinz E (1997) Desaturase multigene families of Brassica napus arose through genome duplication. Theor Appl Genet 94:583-591

Smith MA, Moon H, Chowrira G, Kunst L (2003) Heterologous expression of a fatty acid hydroxylase gene in developing seeds of Arabidopsis. Planta 217:507-516

Smooker A, Wells R, Morgan C, Beaudoin F, Cho K, Fraser F, Bancroft I (2011) The identification and mapping of candidate genes and QTL involved in the fatty acid desaturation pathway in Brassica napus. Theor Appl Genet 122: $1075-1090$

Stemple DL (2004) TILLING-a high-throughput harvest for functional genomics. Nat Rev Genet 5(2):5. doi:10.1038/ $\operatorname{nrg} 1273$

Stephenson P, Baker D, Girin T, Perez A, Amoah S, King G, Ostergaard L (2010) A rich TILLING resource for studying gene function in Brassica rapa. BMC Plant Biol 10(1):62

Town CD, Cheung F, Maiti R, Crabtree J, Haas BJ, Wortman JR, Hine EE, Althoff R, Arbogast TS, Tallon LJ, Vigouroux M, Trick M, Bancroft I (2006) Comparative genomics of Brassica oleracea and Arabidopsis thaliana reveal gene loss, fragmentation, and dispersal after polyploidy. Plant Cell 18(6):1348-1359. doi:10.1105/tpc.106.041665

Wang N, Wang Y, Tian F, King GJ, Zhang C, Long Y, Shi L, Meng J (2008) A functional genomics resource for Brassica napus: development of an EMS mutagenized population and discovery of FAE1 point mutations by TILLING. New Phytol 180(4):751-765. doi:10.1111/j. 1469-8137.2008.02619.x
Wang X, Wang H, Wang J, Sun R, Wu J, Liu S, Bai Y, Mun J-H, Bancroft I, Cheng F, Huang S, Li X, Hua W, Wang J, Wang X, Freeling M, Pires JC, Paterson AH, Chalhoub B, Wang B, Hayward A, Sharpe AG, Park B-S, Weisshaar B, Liu B, Li B, Liu B, Tong C, Song C, Duran C, Peng C, Geng C, Koh C, Lin C, Edwards D, Mu D, Shen D, Soumpourou E, Li F, Fraser F, Conant G, Lassalle G, King GJ, Bonnema G, Tang H, Wang H, Belcram H, Zhou H, Hirakawa H, Abe H, Guo H, Wang H, Jin H, Parkin IAP, Batley J, Kim J-S, Just J, Li J, Xu J, Deng J, Kim JA, Li J, Yu J, Meng J, Wang J, Min J, Poulain J, Hatakeyama K, Wu K, Wang L, Fang L, Trick M, Links MG, Zhao M, Jin M, Ramchiary N, Drou N, Berkman PJ, Cai Q, Huang Q, Li R, Tabata S, Cheng S, Zhang S, Zhang S, Huang S, Sato S, Sun S, Kwon S-J, Choi S-R, Lee T-H, Fan W, Zhao X, Tan X, Xu X, Wang Y, Qiu Y, Yin Y, Li Y, Du Y, Liao Y, Lim Y, Narusaka Y, Wang Y, Wang Z, Li Z, Wang Z, Xiong Z, Zhang Z (2011) The genome of the mesopolyploid crop species Brassica rapa. Nat Genet 43(10):1035-1039. http://www.nature.com/ng/ journal/v43/n10/abs/ng.919.html\#supplementary-information

Yang T-J, Kim JS, Kwon S-J, Lim K-B, Choi B-S, Kim J-A, Jin M, Park JY, Lim M-H, Kim H-I, Lim YP, Kang JJ, Hong J-H, Kim C-B, Bhak J, Bancroft I, Park B-S (2006) Sequence-level analysis of the diploidization process in the triplicated FLOWERING LOCUS C region of Brassica rapa. Plant Cell Online 18(6):1339-1347. doi:10.1105/tpc. 105.040535

Yang Q, Fan C, Guo Z, Qin J, Wu J, Li Q, Fu T, Zhou Y (2012a) Identification of FAD2 and FAD3 genes in Brassica napus genome and development of allele-specific markers for high oleic and low linolenic acid contents. Theor Appl Genet 125(4):715-729. doi:10.1007/s00122-012-1863-1

Yang Q, Fan C, Guo Z, Qin J, Wu J, Li Q, Fu T, Zhou Y (2012b) Identification of FAD2 and FAD3 genes in Brassica napus genome and development of allele-specific markers for high oleic and low linolenic acid contents. Theor Appl Genet 125(4):715-729. doi:10.1007/s00122-012-1863-1 